\title{
SOME INEQUALITIES FOR THE NORM AND THE NUMERICAL RADIUS OF LINEAR OPERATORS IN HILBERT SPACES
}

\author{
S. S. DRAGOMIR
}

\begin{abstract}
In this paper various inequalities between the operator norm and its numerical radius are provided. For this purpose, we employ some classical inequalities for vectors in inner product spaces due to Buzano, GoldsteinRyff-Clarke, Dragomir-Sándor and the author.
\end{abstract}

\section{Introduction}

Let $(H ;\langle\cdot, \cdot\rangle)$ be a complex Hilbert space. The numerical range of an operator $T$ is the subset of the complex numbers $\mathbb{C}$ given by $[9$, p. 1]:

$$
W(T)=\{\langle T x, x\rangle, x \in H,\|x\|=1\} .
$$

It is well known (see for instance [9]) that:

(i) The numerical range of an operator is convex (the Toeplitz-Hausdorff theorem);

(ii) The spectrum of an operator is contained in the closure of its numerical range;

(iii) $T$ is self-adjoint if and only if $W$ is real.

The numerical radius $w(T)$ of an operator $T$ on $H$ is defined by [9, p. 8]:

$$
w(T)=\sup \{|\lambda|, \lambda \in W(T)\}=\sup \{|\langle T x, x\rangle|,\|x\|=1\} .
$$

It is well known that $w(\cdot)$ is a norm on the Banach algebra $B(H)$ and the following inequality holds true

$$
w(T) \leq\|T\| \leq 2 w(T), \text { for any } T \in B(H) .
$$

In the previous work [6], the following reverse inequalities have been proved:

$$
(0 \leq)\|T\|-w(T) \leq \frac{1}{2} \cdot \frac{r^{2}}{|\lambda|},
$$

provided that $\lambda \in \mathbb{C} \backslash\{0\}, r>0$ and

$$
\|T-\lambda I\| \leq r .
$$

Received March 17, 2006; revised June 22, 2006.

2000 Mathematics Subject Classification. 47A12.

Key words and phrases. Numerical range, Numerical radius, Bounded linear operators, Hilbert spaces. 
If, in addition $|\lambda|>r$ and (1.5) holds true, then

$$
\left(1-\frac{r^{2}}{|\lambda|^{2}}\right)^{\frac{1}{2}} \leq \frac{w(T)}{\|T\|}(\leq 1),
$$

which provides a refinement of the general inequality

$$
\frac{1}{2} \leq \frac{w(T)}{\|T\|}
$$

in the case when $r$ and $\lambda$ satisfy the assumption $r /|\lambda| \leq \sqrt{3} / 2$.

With the same assumption on $\lambda$ and $r$, i.e. $|\lambda|>r$, we also have the inequality

$$
(0 \leq)\|T\|^{2}-w^{2}(T) \leq \frac{2 r^{2}}{|\lambda|+\sqrt{|\lambda|^{2}-r^{2}}} w(T),
$$

provided (1.5) holds true.

In the same paper, on assuming that $\left(T^{*}-\bar{\varphi} I\right)(\phi I-T)$ is accretive [9, p. 25] (or sufficiently, self-adjoint and nonnegative in the operator order of $B(H)$ ), where $\varphi, \phi \in \mathbb{C}, \phi \neq-\varphi, \varphi$, we have proved the following inequality as well:

$$
(0 \leq)\|T\|-w(T) \leq \frac{1}{4} \cdot \frac{|\phi-\varphi|^{2}}{|\phi+\varphi|} .
$$

If we assume more, i.e., $\operatorname{Re}(\phi \bar{\varphi})>0$ (which implies $\phi \neq-\varphi$ ), then for $T$ as above, we also have:

$$
\frac{2 \sqrt{\operatorname{Re}(\phi \bar{\varphi})}}{|\phi+\varphi|} \leq \frac{w(T)}{\|T\|}(\leq 1)
$$

and

$$
(0 \leq)\|T\|^{2}-w^{2}(T) \leq[|\phi+\varphi|-2 \sqrt{\operatorname{Re}(\phi \bar{\varphi})}] w(T) .
$$

The main aim of this paper is to establish other inequalities between the operator norm and its numerical radius. We employ, amongst others, the Buzano inequality as well as some results for vectors in inner product spaces due to Goldstein-Ryff-Clarke [8], Dragomir-Sándor [7] and Dragomir [5].

\section{The Results}

The following result may be stated as well:

Theorem 1. Let $(H ;\langle\cdot, \cdot\rangle)$ be a Hilbert space and $T: H \rightarrow H$ a bounded linear operator on $H$. Then

$$
w^{2}(T) \leq \frac{1}{2}\left[w\left(T^{2}\right)+\|T\|^{2}\right] .
$$


The constant $\frac{1}{2}$ is best possible in (2.1).

Proof. We need the following refinement of Schwarz's inequality obtained by the author in 1985 [2, Theorem 2] (see also [7] and [5]):

$$
\|a\|\|b\| \geq|\langle a, b\rangle-\langle a, e\rangle\langle e, b\rangle|+|\langle a, e\rangle\langle e, b\rangle| \geq|\langle a, b\rangle|,
$$

provided $a, b, e$ are vectors in $H$ and $\|e\|=1$.

Observing that

$$
|\langle a, b\rangle-\langle a, e\rangle\langle e, b\rangle| \geq|\langle a, e\rangle\langle e, b\rangle|-|\langle a, b\rangle|,
$$

hence by the first inequality in (2.2) we deduce

$$
\frac{1}{2}(\|a\|\|b\|+|\langle a, b\rangle|) \geq|\langle a, e\rangle\langle e, b\rangle| .
$$

This inequality was obtained in a different way earlier by M.L. Buzano in [1].

Now, choose in (2.3), $e=x,\|x\|=1, a=T x$ and $b=T^{*} x$ to get

$$
\frac{1}{2}\left(\|T x\|\left\|T^{*} x\right\|+\left|\left\langle T^{2} x, x\right\rangle\right|\right) \geq|\langle T x, x\rangle|^{2}
$$

for any $x \in H,\|x\|=1$.

Taking the supremum in (2.4) over $x \in H,\|x\|=1$, we deduce the desired inequality (2.1).

Now, if we assume that (2.1) holds with a constant $C>0$, i.e.,

$$
w^{2}(T) \leq C\left[w\left(T^{2}\right)+\|T\|^{2}\right]
$$

for any $T \in B(H)$, then if we choose $T$ a normal operator and use the fact that for normal operators we have $w(T)=\|T\|$ and $w\left(T^{2}\right)=\left\|T^{2}\right\|=\|T\|^{2}$, then by (2.5) we deduce that $2 C \geq 1$ which proves the sharpness of the constant.

Remark 1. From the above result (2.1) we obviously have

$$
w(T) \leq\left\{\frac{1}{2}\left[w\left(T^{2}\right)+\|T\|^{2}\right]\right\}^{1 / 2} \leq\left\{\frac{1}{2}\left(\left\|T^{2}\right\|+\|T\|^{2}\right)\right\}^{1 / 2} \leq\|T\|
$$

and

$$
w(T) \leq\left\{\frac{1}{2}\left[w\left(T^{2}\right)+\|T\|^{2}\right]\right\}^{1 / 2} \leq\left\{\frac{1}{2}\left(w^{2}(T)+\|T\|^{2}\right)\right\}^{1 / 2} \leq\|T\|,
$$

that provide refinements for the first inequality in (1.3).

The following result may be stated.

Theorem 2. Let $T: H \rightarrow H$ be a nonzero bounded linear operator on the Hilbert space $H$, then

$$
\|T\| \leq w(T)+\frac{1}{2}\|T\|^{-1}\|T-\| T\|\cdot I\|^{2} .
$$


Proof. We use the following inequality for vectors in inner product spaces due to Goldstein, Ryff and Clarke [8]:

$$
\|a\|^{2 r}+\|b\|^{2 r}-2\|a\|^{r}\|b\|^{r} \frac{\operatorname{Re}\langle a, b\rangle}{\|a\|\|b\|}
$$

$$
\leq \begin{cases}r^{2}\|a\|^{2 r-2}\|a-b\|^{2} & \text { if } r \geq 1, \\ \|b\|^{2 r-2}\|a-b\|^{2} & \text { if } r<1,\end{cases}
$$

provided $r \in \mathbb{R}$ and $a, b \in H$ with $\|a\| \geq\|b\|$.

Now, let $x \in H$ with $\|x\|=1$. From the hypothesis of the theorem, we have that $\|T x\| \leq$ $\|T\|\|x\|$ and applying (2.9) for the choices $a=\|T\| x,\|x\|=1, b=T x$, we get

$$
\|T x\|^{2 r}+\|T\|^{2 r}-2\|T x\|^{r-1}\|T\|^{r}|\langle T x, x\rangle| \leq r^{2}\|T\|^{2 r-2}\|T x-\| T\|x\|^{2}
$$

for any $x \in H,\|x\|=1$ and $r \geq 1$.

Taking the supremum in (2.10) over $x \in H,\|x\|=1$, and doing the necessary calculations, we deduce the desired inequality (2.8).

The following result may be stated as well:

Theorem 3. Let $T: H \rightarrow H$ be a bounded linear operator on the Hilbert space $(H,\langle\cdot, \cdot\rangle)$. Then for any $\alpha \in[0,1]$ and $t \in \mathbb{R}$ one has the inequality:

$$
\|T\|^{2} \leq\left[(1-\alpha)^{2}+\alpha^{2}\right] w^{2}(T)+\alpha\|T-t I\|^{2}+(1-\alpha)\|T-i t I\|^{2} .
$$

Proof. We use the following inequality obtained by the author in [5]:

$$
\begin{array}{r}
{\left[\alpha\|t b-a\|^{2}+(1-\alpha)\|i t b-a\|^{2}\right]\|b\|^{2}} \\
\geq\|a\|^{2}\|b\|^{2}-[(1-\alpha) \operatorname{Im}\langle a, b\rangle+\alpha \operatorname{Re}\langle a, b\rangle]^{2}(\geq 0)
\end{array}
$$

to get:

$$
\begin{aligned}
\|a\|^{2}\|b\|^{2} \leq & {[(1-\alpha) \operatorname{Im}\langle a, b\rangle+\alpha \operatorname{Re}\langle a, b\rangle]^{2} } \\
& +\left[\alpha\|t b-a\|^{2}+(1-\alpha)\|i t b-a\|^{2}\right]\|b\|^{2} \\
\leq & {\left[(1-\alpha)^{2}+\alpha^{2}\right]|\langle a, b\rangle|^{2} } \\
& \quad+\left[\alpha\|t b-a\|^{2}+(1-\alpha)\|i t b-a\|^{2}\right]\|b\|^{2}
\end{aligned}
$$

for any $a, b \in H, \alpha \in[0,1]$ and $t \in \mathbb{R}$.

Choosing in (2.12) $a=T x, b=x, x \in H,\|x\|=1$, we get

$$
\|T x\|^{2} \leq\left[(1-\alpha)^{2}+\alpha^{2}\right]|\langle T x, x\rangle|^{2}
$$

$$
+\alpha\|t x-T x\|^{2}+(1-\alpha)\|i t x-T x\|^{2} .
$$


Finally, taking the supremum over $x \in H,\|x\|=1$ in (2.13), we deduce the desired result.

The following particular cases may be of interest.

Corollary 1. For any $T$ a bounded linear operator on $H$, one has:

$$
(0 \leq)\|T\|^{2}-w^{2}(T) \leq\left\{\begin{array}{l}
\inf _{t \in \mathbb{R}}\|T-t I\|^{2} \\
\inf _{t \in \mathbb{R}}\|T-i t I\|^{2}
\end{array}\right.
$$

and

$$
\|T\|^{2} \leq \frac{1}{2} w^{2}(T)+\frac{1}{2} \inf _{t \in \mathbb{R}}\left[\|T-t I\|^{2}+\|T-i t I\|^{2}\right] .
$$

Remark 2. The inequality (2.14) can in fact be improved taking into account that for any $a, b \in H, b \neq 0$, (see for instance [3]) the bound

$$
\inf _{\lambda \in \mathbb{C}}\|a-\lambda b\|^{2}=\frac{\|a\|^{2}\|b\|^{2}-|\langle a, b\rangle|^{2}}{\|b\|^{2}}
$$

actually implies that

$$
\|a\|^{2}\|b\|^{2}-|\langle a, b\rangle|^{2} \leq\|b\|^{2}\|a-\lambda b\|^{2}
$$

for any $a, b \in H$ and $\lambda \in \mathbb{C}$.

Now if in (2.16) we choose $a=T x, b=x, x \in H,\|x\|=1$, then we obtain

$$
\|T x\|^{2}-|\langle T x, x\rangle|^{2} \leq\|T x-\lambda x\|^{2}
$$

for any $\lambda \in \mathbb{C}$, which, by taking the supremum over $x \in H,\|x\|=1$, implies that

$$
(0 \leq)\|T\|^{2}-w^{2}(T) \leq \inf _{\lambda \in \mathbb{C}}\|T-\lambda I\|^{2}
$$

If $T$ and $\phi, \varphi \in \mathbb{C}$ are such that $\left(T^{*}-\bar{\varphi} I\right)(\phi I-T)$ is accretive, or, equivalently

$$
\left\|T-\frac{\phi+\varphi}{2} \cdot I\right\| \leq \frac{1}{2}|\phi-\varphi|,
$$

then, by (2.18) for $\lambda=\frac{\phi+\varphi}{2}$, we obtain

$$
(0 \leq)\|T\|^{2}-w^{2}(T) \leq \frac{1}{4}|\phi-\varphi|^{2}
$$

which is an inequality of interest and related to the ones stated in the introduction.

Remark 3. If we take $a=x, b=T x$ in (2.16), then we obtain

$$
\|T x\|^{2} \leq|\langle T x, x\rangle|^{2}+\|T x\|^{2}\|x-\mu T x\|^{2}
$$


for any $x \in H,\|x\|=1$ and $\mu \in \mathbb{C}$. Now, if we take the supremum over $x \in H,\|x\|=1$ in (2.21), then we get

$$
(0 \leq)\|T\|^{2}-w^{2}(T) \leq\|T\|^{2} \inf _{\mu \in \mathbb{C}}\|I-\mu T\|^{2} .
$$

We also remark that

$$
\|T\|^{2} \inf _{\mu \in \mathbb{C}}\|I-\mu T\|^{2} \leq|\mu|^{2}\|T\|^{2}\left\|T-\frac{1}{\mu} \cdot I\right\|^{2}, \mu \in \mathbb{C} \backslash\{0\}
$$

which for $\mu=\frac{2}{\phi+\varphi}, \phi \neq-\varphi$, gives the inequality

$$
(0 \leq)\|T\|^{2}-w^{2}(T) \leq\|T\|^{2} \cdot\left|\frac{\phi-\varphi}{\phi+\varphi}\right|^{2},
$$

provided $\left(T^{*}-\bar{\varphi} I\right)(\phi I-T)$ is accretive, or, equivalently $\left\|T-\frac{\phi+\varphi}{2} \cdot I\right\| \leq \frac{1}{2}|\phi-\varphi|$.

This is another interesting inequality related to the ones from the introduction.

Finally and from a different view point we may state:

Theorem 4. Let $T: H \rightarrow H$ be a bounded linear operator on $H$. If $p \geq 2$, then:

$$
\|T\|^{p} \leq \frac{1}{4}\left(\|T+\| T\|\cdot I\|^{p}+\|T-\| T\|\cdot I\|^{p}\right) .
$$

Proof. We use the following inequality obtained by Dragomir and Sándor in [7]:

$$
\|a+b\|^{p}+\|a-b\|^{p} \geq 2\left(\|a\|^{p}+\|b\|^{p}\right) \text { for any } a, b \in H \text { and } p \geq 2 .
$$

Now, if we choose $a=T x, b=\|T\| x$, then we get

$$
\|T x+\| T\|x\|^{p}+\|T x-\| T\|x\|^{p} \geq 2\left(\|T x\|^{p}+\|T\|^{p}\right)
$$

for any $x \in H,\|x\|=1$.

Taking the supremum in (2.26) over $x \in H,\|x\|=1$, we get the desired result (2.24).

Remark 4. For $p=2$, we have the simpler result:

$$
\|T\|^{2} \leq \frac{1}{4}\left(\|T+\| T\|\cdot I\|^{2}+\|T-\| T\|\cdot I\|^{2}\right) .
$$

This can easily be obtained from the parallelogram identity as well.

\section{Acknowledgement}

The author would like to thank the anonymous referee for his/her valuable comments that have been implemented in the final version of the paper. 


\section{References}

[1] M. L. Buzano, Generalizzazione della disiguaglianza di Cauchy-Schwaz (Italian), Rend. Sem. Mat. Univ. e Politech. Torino, 31 (1971/73), 405-409 (1974).

[2] S. S. Dragomir, Some refinements of Schwarz inequality, Simposional de Matematică şi Aplicaţii, Polytechnical Institute Timişoara, Romania, 1-2 Nov., 1985, 13-16. ZBL 0594:46018.

[3] S. S. Dragomir, Some Grüss type inequalities in inner product spaces, J. Ineq. Pure \& Appl. Math., 4(2003), Art. 42. [ONLINE http://jipam.vu.edu.au/article.php?sid=280].

[4] S. S. Dragomir, A potpourri of Schwarz related inequalities in inner product spaces (I), J. Ineq. Pure \& Appl. Math., 6(2005), Art. 59. [ONLINE http://jipam.vu.edu.au/article.php?sid=532].

[5] S. S. Dragomir, A potpourri of Schwarz related inequalities in inner product spaces (II), J. Ineq. Pure \& Appl. Math., 7(2006), Art. 14. [ONLINE http://jipam.vu.edu.au/article.php?sid=619].

[6] S. S. Dragomir, Reverse inequalities for the numerical radius of linear operators in Hilbert spaces, Bull. Austral. Math. Soc., 73(2006), 255-262. Preprint available on line at RGMIA Res. Rep. Coll. 8(2005), Article 9, [ONLINE http://rgmia.vu.edu.au/v8(E).html].

[7] S.S. Dragomir and J. Sándor, Some inequalities in prehilbertian spaces, Studia Univ. "Babeş-Bolyai”Mathematica, 32(1987), 71-78.

[8] A. Goldstein, J. V. Ryff and L. E. Clarke, Problem 5473, Amer. Math. Monthly, 75(1968), 309.

[9] K. E. Gustafson and D. K. M. Rao, Numerical Range, Springer-Verlag, New York, Inc., 1997.

School of Computer Science and Mathematics, Victoria University, PO Box 14428, Melbourne City, Victoria 8001 , Australia.

E-mail: sever.dragomir@vu.edu.au

Homepage:http://www.staff.vu.edu.au/rgmia/dragomir/ 